\title{
Comparison of measures for haplotype similarity
}

\author{
Vivien Marquard ${ }^{\dagger}$, Lars Beckmann ${ }^{\dagger 1}$, Justo L Bermejo ${ }^{2}$, Christine Fischer $^{3}$ \\ and Jenny Chang-Claude*1
}

Address: ${ }^{1}$ Cancer Epidemiology, German Cancer Research Center DKFZ, Im Neuenheimer Feld 280, 69120 Heidelberg, Germany, ${ }^{2}$ Molecular Genetic Epidemiology, German Cancer Research Center DKFZ, Im Neuenheimer Feld 280, 69120 Heidelberg, Germany and ${ }^{3}$ Institute of Human Genetics, University of Heidelberg, Im Neuenheimer Feld 366, 69120 Heidelberg, Germany

Email: Vivien Marquard - v.marquard@dkfz-heidelberg.de; Lars Beckmann - l.beckmann@dkfz-heidelberg.de; Justo L Bermejo - j.lorenzo@dkfzheidelberg.de; Christine Fischer - cfischer@uni-hd.de; Jenny Chang-Claude* - j.chang-claude@dkfz-heidelberg.de

* Corresponding author †Equal contributors

from Genetic Analysis Workshop 15

St. Pete Beach, Florida, USA. I I-I5 November 2006

Published: 18 December 2007

BMC Proceedings 2007, I (Suppl I):SI28

This article is available from: http://www.biomedcentral.com/I753-656I/I/SI/SI 28

(c) 2007 Marquard et al; licensee BioMed Central Ltd.

This is an open access article distributed under the terms of the Creative Commons Attribution License (http://creativecommons.org/licenses/by/2.0), which permits unrestricted use, distribution, and reproduction in any medium, provided the original work is properly cited.

\begin{abstract}
Measuring the association of haplotype similarities with phenotype similarities has been used to develop statistical tests of genetic association. Previously, we applied the general approach of Mantel statistics to correlate genetic and phenotype similarity, where genetic similarity was defined by the number of intervals flanked by markers identical by state for pairs of haplotypes. Here we investigated in the case-control study design the effect on power of the Mantel statistics for five different measures of genetic similarity based on haplotypes: I) the number of shared intervals, 2) the physical length of the shared intervals, 3) the genetic length of the shared intervals in centimorgans, 4) the genetic length of the shared intervals in linkage disequilibrium units (LDU) and 5) Yu's measure that attaches more weight to the sharing of rare than common alleles. With prior knowledge of the answers of Genetic Analysis Workshop 15 Problem 3, we analyzed the simulated data sets in two genomic regions surrounding the disease loci on chromosomes 6 and 18 . For the dense map on chromosome 6 , all methods showed a very high power of comparable magnitude. For chromosome 18, we observed a power between $19 \%$ and $99 \%$ at the pointwise $5 \%$ significance level using 1000 cases and 1000 controls for all methods except Yu's measure. While it yielded a much lower power, Yu's measure had $80 \%$ power around the disease locus.
\end{abstract}

\section{Background}

The potential value of haplotypes in the mapping of complex traits has attracted widespread interest. A convenient approach to incorporating haplotype information is the search for shared chromosomal segments. Haplotypesharing approaches are based on the assumption that, in the vicinity of a predisposing mutation, haplotypes carry- ing this mutation are more similar than haplotypes without the mutation. The expectation is that the case haplotypes share significantly longer stretches of DNA identically by descent (IBD) around the mutation. Thus, the first proposed measure of similarity between haplotypes for gene mapping was the number of intervals flanked by the same marker alleles, i.e., by markers iden- 
tical by state (IBS) [1]. However, this approach does not take into account marker spacing and linkage disequilibrium (LD). This study investigated whether alternative haplotype similarity measures improve the power of haplotype-sharing analysis. We weighted the intervals by their physical length, and by their genetic length measured in centimorgans and in linkage disequilibrium units (LDUs). Furthermore, we studied an approach that gives more weights to the sharing for rare marker alleles than for common ones.

To analyze the dependence of power on the different similarity measures, we used a previously developed Mantel statistic to correlate genetic and phenotypic similarity [2]. We used the simulated data sets of Genetic Analysis Workshop 15 (GAW15) Problem 3 in two genomic regions for a population-based case-control scenario.

\section{Methods}

\section{Simulated data sets}

From the simulated rheumatoid arthritis data set consisting of 1500 families with two affected children and 2000 unrelated controls (Problem 3), the first affected child from each of the first 1000 families was chosen to constitute the case group. For each replication the cases were matched by sex with 1000 controls. With prior knowledge of the disease-causing loci, 21 SNPs (3427 to 3447) including Locus $\mathrm{C}$ of the high-density scan of chromosome 6 were extracted. In addition, 20 SNPs (260 to 279) from chromosome 18 around Locus $\mathrm{E}$ were chosen.

Because of the known strong effect of Locus C, smaller samples each consisting of 50 or 100 cases and controls were used for the analysis of chromosome 6 . Females and males were analyzed separately because of the known gender-specific interaction between Locus $\mathrm{C}$ and the Disease Locus DR. Data on chromosome 18 was analyzed using samples of 500 or 1000 cases and controls for both sexes combined. The haplotypes used for analysis were provided by GAW15.

\section{Measures of haplotype similarity $\mathrm{L}_{\mathrm{ij}}(\mathbf{x})$}

We applied four different measures of haplotype similarity based on the number of shared intervals, i.e., number of intervals surrounding a marker that are flanked by markers with the same alleles (IBS). Modified versions of these four measures were also employed to take into account the sharing of a single marker and the unobserved regions between the examined markers beyond the shared region. The first and common measure $N$ counts the number of shared intervals in the vicinity of a specific marker. The modified version of this measure, $\mathrm{N+}$, corresponds to $N+1$. For the next three measures, $N$ was weighted with the physical, $\mathrm{KB}$, or genetic length, $\mathrm{CM}$, or LDU, between the first and the last shared markers in (kilo)base pairs, centimorgans, or LDUs, respectively. LDUs were introduced by Morton et al. as a genetic distance based on the observed haplotype frequencies [3]. Maniatis et al. showed that the use of LDUs might improve the power of single-point linkage analysis and the power to identify disease-causing variants [4]. The software LDMAP [4] has been used to determine LDUs of the chosen markers. For the modified versions, $\mathrm{KB}+, \mathrm{CM}+$, and LDU+, the half of the distance both before the first shared marker and after the last shared marker were added. When either the first or the last of all investigated markers was involved, then half of the distance to the second and to the penultimate marker was used as a proxy, respectively. We also studied the measure proposed by $\mathrm{Yu}$ et al. [5], which gives greater weights to the sharing for rare marker alleles than to that for common alleles. The weights are determined as the probability of particular alleles at the specific marker, conditional on the surrounding alleles and are estimated from the allele frequencies of control haplotypes.

\section{Exploratory analysis}

Kruskal's nonmetric multidimensional scaling (MDS) was used to explore the resemblance among the different similarity measures [6]. MDS performs a minimizing algorithm based on the stress value, a least square estimator that assesses the dimensionality of the data using the observed and estimated distances. The smaller the stress value, the better the fit, with stress values between 0 and 2.5 indicating an excellent goodness of fit. After dimensionality assessment, a graphical representation of the data permitted the investigation of resemblance among similarity measures.

\section{Mantel statistics using haplotype sharing and power analysis}

The haplotype-based Mantel statistic correlates the haplotype similarity $L_{i j}$ for every marker $x$, where $i$ and $j$ are two haplotypes, and the phenotypic similarity $Y_{s_{i} s_{j}}$ from two individuals $s_{i}$ and $s_{j}$ corresponding to the haplotypes $i$ and $j$ [2]. The phenotypic similarity $Y_{s_{i} s_{j}}$ is defined as the mean corrected product $Y_{s_{i} s_{j}}=\left(Y_{s_{i}}-\mu\right)\left(Y_{s_{j}}-\mu\right)$, where $\mu$ denotes the expectation of the phenotype in the sample, i.e., $\mu=0.5$, while a case was coded as 1 and a control as 0 . Thus, the defined statistic is the sum of the cross products of $L_{i j}(x)$ and $Y_{s_{i} s_{j}}$ :

$$
M(x)=\sum_{i<j} L_{i j}(x) Y_{s_{i} s_{j}}
$$


Statistical significance was assessed via a Monte Carlo permutation. The empirical null distribution, i.e., the distribution in which the genetic and the phenotypic similarity were independently distributed, was estimated by permuting the phenotype 1000 times while keeping together the two haplotypes derived from an individual. The empirical $p$-value was derived by comparing the observed statistic against the empirical distribution.

The different haplotype similarity measures were calculated for each replication $r=1, \ldots, 100$ for both chromosomes and employed to investigate the power of the Mantel statistics to map the disease locus. The power was estimated as the number of replications with a significant test result ( $p$-value less than $\alpha=0.05$ ) divided by 100 , the total number of replications.

The data management, the calculation of the similarity measures as well as the Mantel statistics were performed in the $\mathrm{R}$ programming language.

\section{Results}

The exploratory analysis using MDS yielded values of stress $=0.01$ for chromosome 6 and stress $=0.80$ for chromosome 18, both indicating an excellent goodness of fit. Therefore, a one-dimensional representation was suffi-
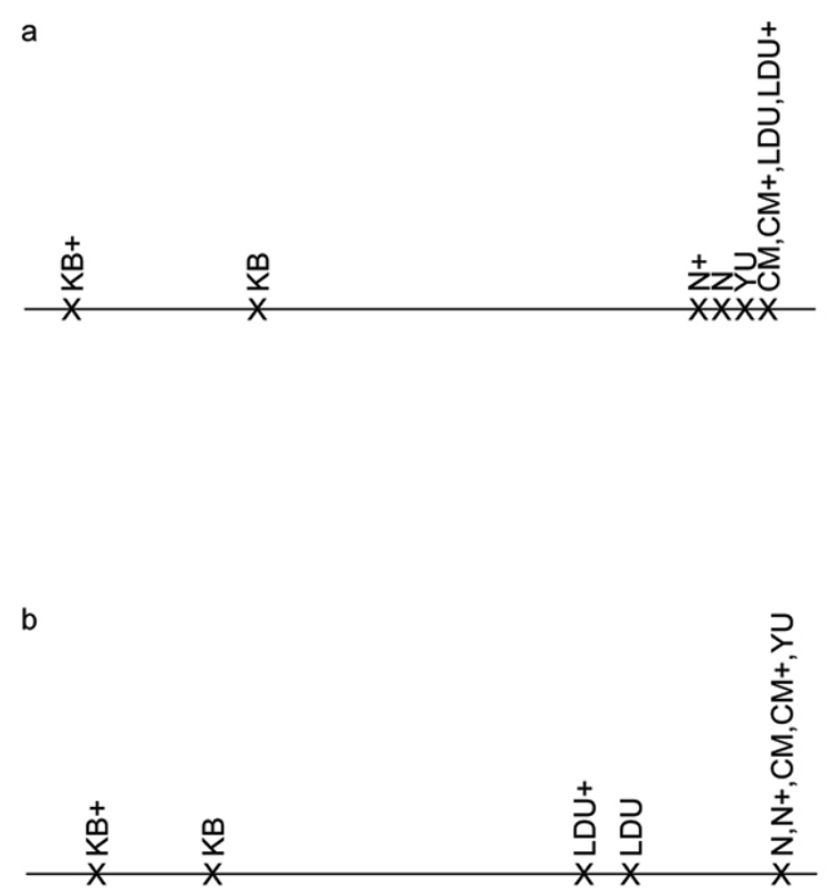

\section{Figure I}

Results from MDS analyses. Results from MDS analyses for chromosome 6 (a) and chromosome 18 (b). The distances among the abbreviations on the axis reflect the resemblance among the similarity measures. cient to describe the resemblance between the similarity measures (Fig. 1a, b). For chromosome 6, the results showed a clear difference between physical distances (in particular $\mathrm{KB}$ and $\mathrm{KB}+$ ) and the other similarity measures. The number of shared intervals $(\mathrm{N}, \mathrm{N}+)$ and $\mathrm{YU}$ were slightly different from genetic distances $(\mathrm{CM}, \mathrm{CM}+\mathrm{LDU}$, and LDU+), which were undistinguishable (Fig. 1a). For chromosome 18, three clusters of similarity measures were identified by MDS: i) $\mathrm{KB}, \mathrm{KB}+$, ii) $\mathrm{LDU}, \mathrm{LDU}+$, and iii) the remaining measures $\mathrm{N}, \mathrm{N}+, \mathrm{CM}, \mathrm{CM}$, and $\mathrm{YU}$ (Fig. 1b). The relationship between the genetic distances measured in centimorgans and LDUs is shown in Figure 2.
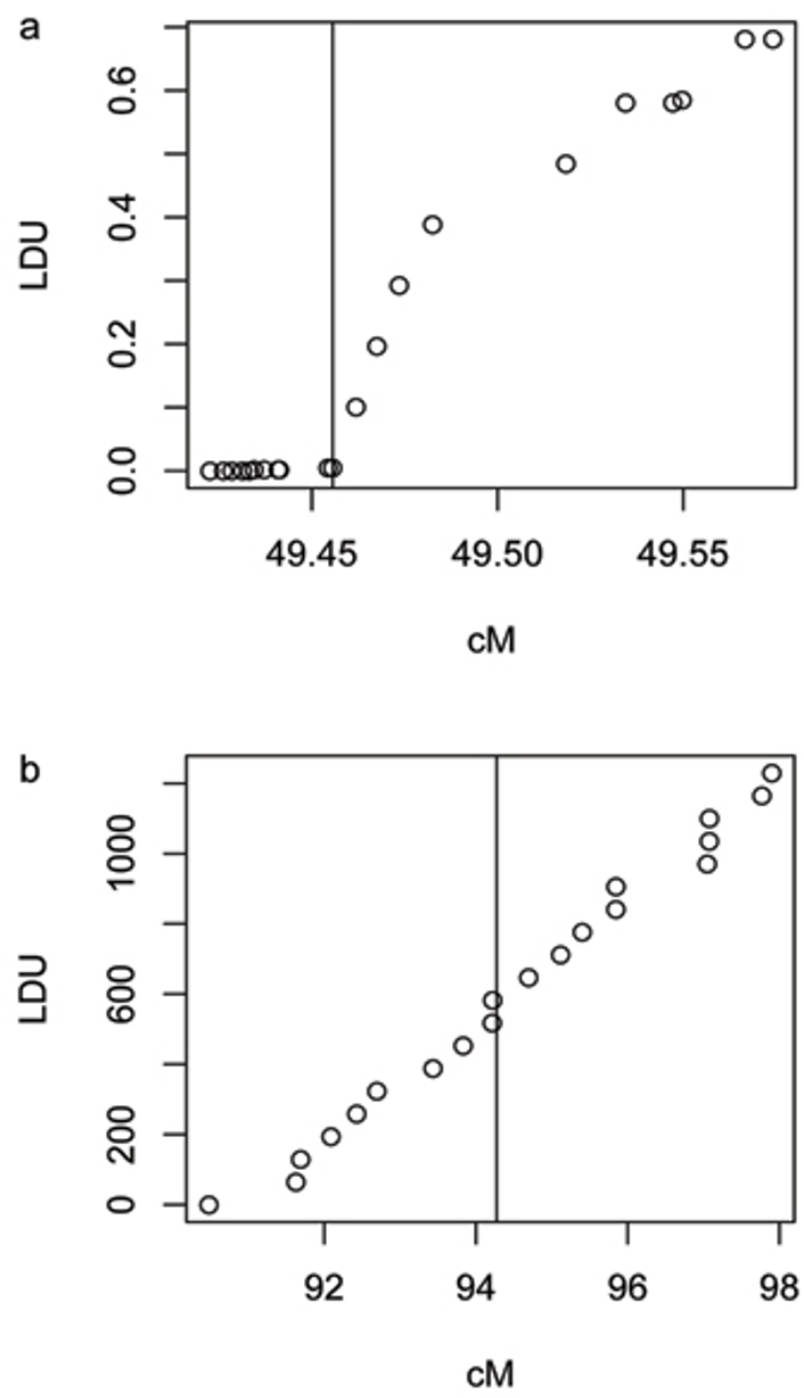

\section{Figure 2}

Relationship between the genetic distance measures. Comparison of the genetic distances in LDU with the genetic distances in centimorgans for chromosome 6 (a) and chromosome 18 (b). Vertical line denotes the location of the disease locus. 
a
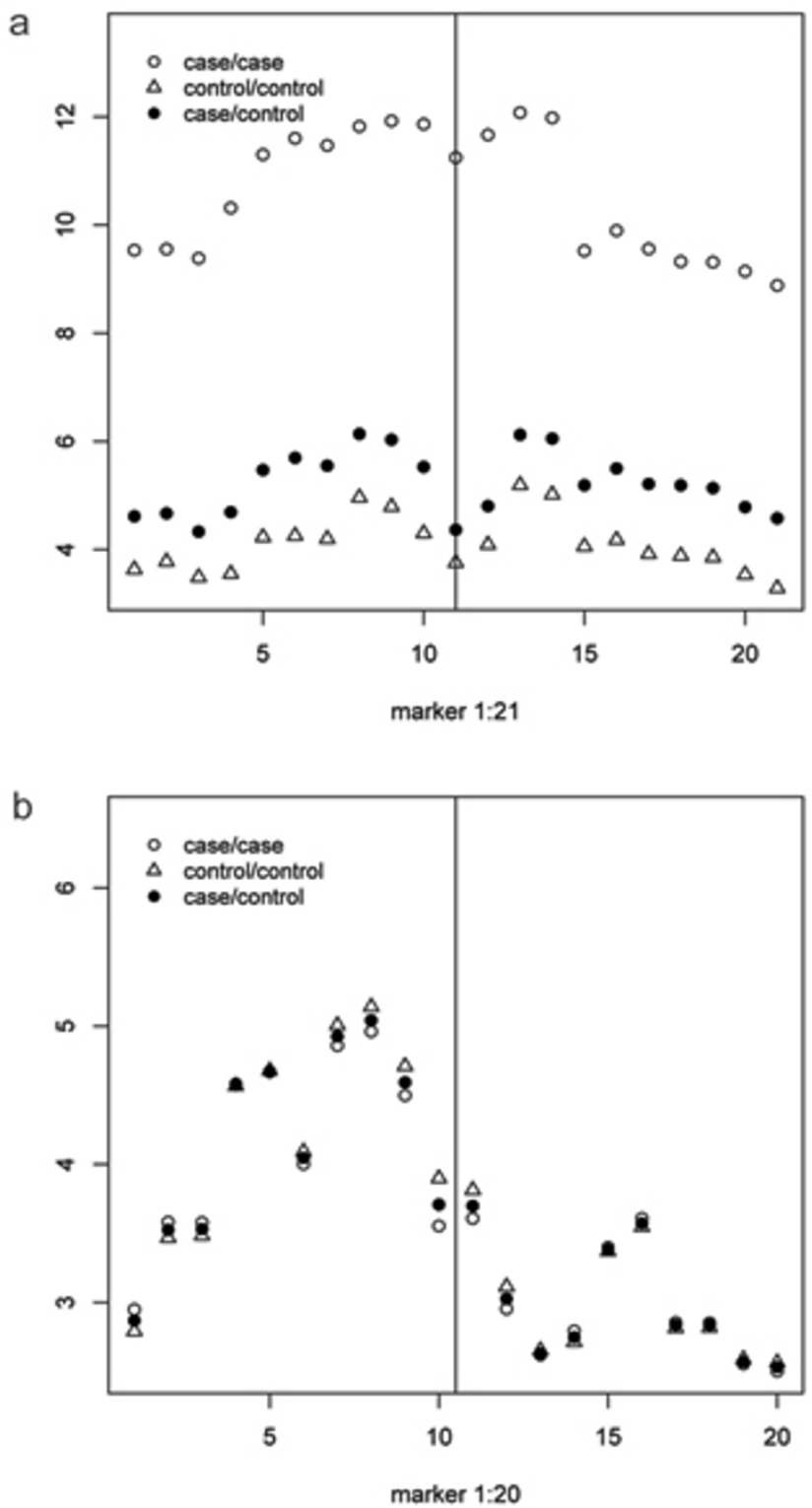

Figure 3

Mean sharing. Case-case, case-control, and control-control haplotype pairs, mean sharing for similarity measure $N$ for every examined marker on chromosome 6 (a) and chromosome 18 (b). Vertical lines denote the location of the disease locus.

On chromosome 6, the genetic distances in centimorgans among the first ten markers were small, but they were null when measured in LDUs, indicating complete LD in this region (Fig. 2a). On chromosome 18, LDUs and centimorgan distances were approximately linearly correlated (Fig. 2b). The markers covered a larger region on chromosome 18 (4000 kb) than on chromosome 6 (300 kb), but gaps can be observed on the genetic scales for both chromosomal regions. a

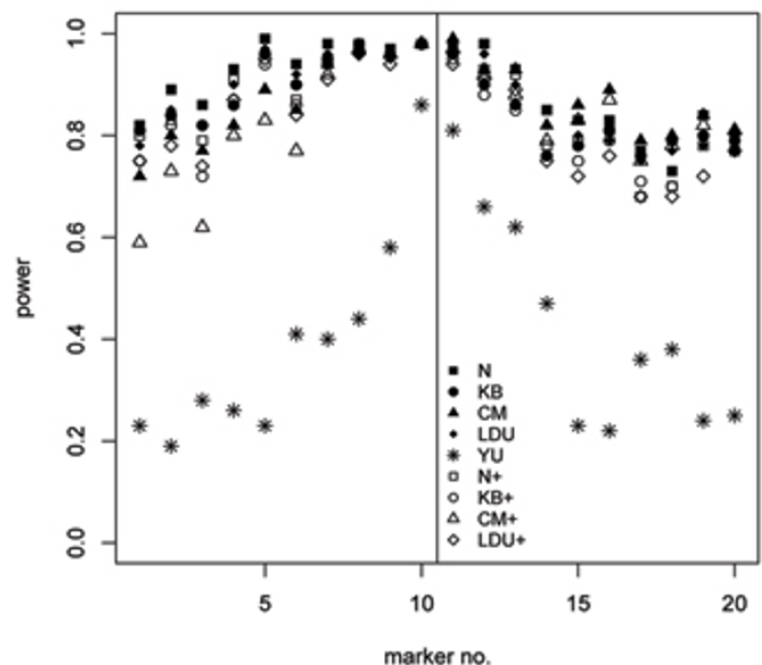

b

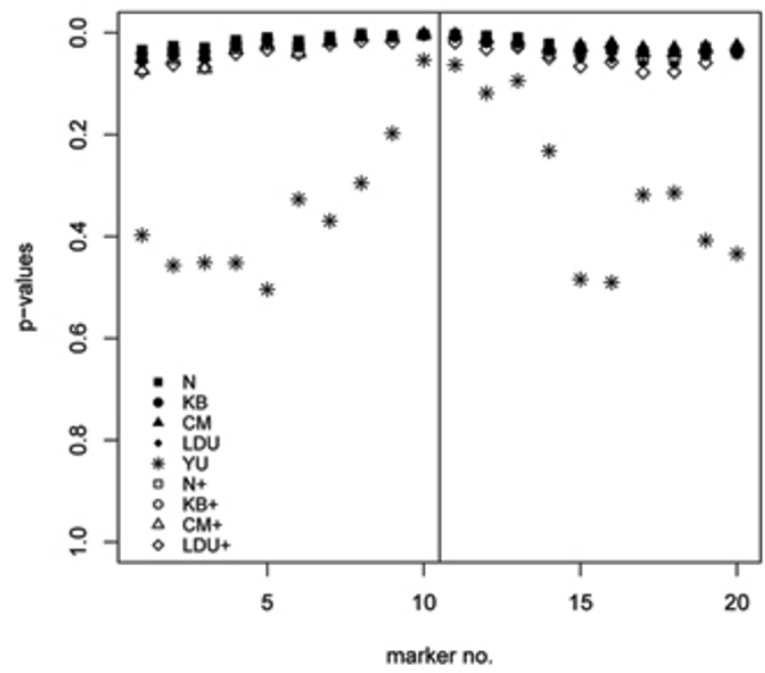

Figure 4

Power and $p$-values. Comparison of power (a) and $p$-values (b) for the nine haplotype similarity measures for each examined marker on chromosome 18 with a sample size of 1000. Vertical lines denote the location of the disease locus.

The measure of haplotype similarity $N$ between haplotypes $i$ and $j$ at marker position $x, L_{i j}(x)$, was calculated for case-case, case-control, and control-control haplotype pairs. Figure 3 a displays the mean sharing for these groups at every marker position $x$ on chromosome 6 . For each similarity measures employed, the mean sharing among case-case pairs was larger than for case-control or controlcontrol pairs, indicating that cases share longer stretches of haplotypes than controls in the vicinity of the susceptibility gene. However, for chromosome 18 this pattern was not observed (Fig. 3b).

For males, the power to identify Locus $\mathrm{C}$ on chromosome 6 was $100 \%$ for all measures in both investigated sample 
Table I: Power

\begin{tabular}{llllllllll}
\hline Marker no ${ }^{\mathrm{a}}$ & $\mathrm{N}$ & $\mathrm{N}+$ & $\mathrm{KB}$ & $\mathrm{KB}+$ & $\mathrm{CM}$ & $\mathrm{CM}+$ & LDU & LDU+ & YU \\
\hline 260 & 0.82 & 0.8 & 0.81 & 0.75 & 0.72 & 0.59 & 0.78 & 0.75 & 0.23 \\
261 & 0.89 & 0.83 & 0.84 & 0.82 & 0.8 & 0.73 & 0.85 & 0.78 & 0.19 \\
262 & 0.86 & 0.79 & 0.82 & 0.72 & 0.77 & 0.62 & 0.82 & 0.74 & 0.28 \\
263 & 0.93 & 0.91 & 0.86 & 0.87 & 0.82 & 0.8 & 0.9 & 0.87 & 0.26 \\
264 & 0.99 & 0.99 & 0.96 & 0.94 & 0.89 & 0.83 & 0.97 & 0.95 & 0.23 \\
265 & 0.94 & 0.87 & 0.9 & 0.86 & 0.85 & 0.77 & 0.92 & 0.84 & 0.41 \\
266 & 0.98 & 0.95 & 0.95 & 0.94 & 0.94 & 0.92 & 0.96 & 0.91 & 0.4 \\
267 & 0.98 & 0.98 & 0.97 & 0.98 & 0.97 & 0.97 & 0.96 & 0.98 & 0.44 \\
268 & 0.97 & 0.96 & 0.96 & 0.96 & 0.96 & 0.96 & 0.95 & 0.94 & 0.58 \\
269 & $\mathbf{0 . 9 8}$ & $\mathbf{0 . 9 8}$ & $\mathbf{0 . 9 8}$ & $\mathbf{0 . 9 8}$ & $\mathbf{0 . 9 8}$ & $\mathbf{0 . 9 8}$ & $\mathbf{0 . 9 8}$ & $\mathbf{0 . 9 8}$ & $\mathbf{0 . 8 6}$ \\
$\mathbf{2 7 0}$ & $\mathbf{0 . 9 8}$ & $\mathbf{0 . 9 7}$ & $\mathbf{0 . 9 6}$ & $\mathbf{0 . 9 5}$ & $\mathbf{0 . 9 9}$ & $\mathbf{0 . 9 7}$ & $\mathbf{0 . 9 6}$ & $\mathbf{0 . 9 4}$ & $\mathbf{0 . 8 1}$ \\
271 & 0.98 & 0.93 & 0.9 & 0.88 & 0.93 & 0.92 & 0.96 & 0.91 & 0.66 \\
272 & 0.93 & 0.92 & 0.86 & 0.85 & 0.93 & 0.88 & 0.9 & 0.89 & 0.62 \\
273 & 0.85 & 0.78 & 0.76 & 0.76 & 0.82 & 0.79 & 0.82 & 0.75 & 0.47 \\
274 & 0.83 & 0.79 & 0.78 & 0.75 & 0.86 & 0.83 & 0.8 & 0.72 & 0.23 \\
275 & 0.83 & 0.81 & 0.81 & 0.79 & 0.89 & 0.87 & 0.79 & 0.76 & 0.22 \\
276 & 0.77 & 0.68 & 0.76 & 0.71 & 0.79 & 0.75 & 0.75 & 0.68 & 0.36 \\
277 & 0.73 & 0.7 & 0.79 & 0.7 & 0.8 & 0.78 & 0.77 & 0.68 & 0.38 \\
278 & 0.84 & 0.78 & 0.8 & 0.8 & 0.84 & 0.82 & 0.78 & 0.72 & 0.24 \\
279 & 0.8 & 0.77 & 0.79 & 0.79 & 0.81 & 0.81 & 0.77 & 0.77 & 0.25 \\
\hline
\end{tabular}

aPower comparison for the nine haplotype similarity measures for each examined marker on chromosome 18, where markers 269 and 270 are the flanking markers of the disease locus.

sizes. For females, the power was $100 \%$ for all measures except LDU and LDU+ using sample sizes of 50 and 100 (data not shown). In contrast, the power to detect Locus $\mathrm{E}$ on chromosome 18 did not exceed $43 \%$ using a sample size of 500, and Yu's measure had a lower power than the other similarity measures for all examined markers (data not shown). However, with a sample size of 1000, all measures, except that of YU, yielded a high power of around $98 \%$ at the markers around the disease locus, but showed different decay of power at distal markers. Whereas Yu's measure showed a power of $80 \%$ around the disease locus ( $15 \%$ less than the other similarity measures), it had a substantially low power of around $20 \%$ at the distal markers (40-60\% less than the other measures) (Table 1 and Fig. 4a). The pronounced peak of the power using Yu's measure corresponded with low p-values (Fig. $4 b)$.

\section{Discussion}

MDS results indicated that for both regions the similarity measures based on physical distances, $\mathrm{KB}$ and $\mathrm{KB}+$, are clearly distinguishable from the other investigated measures. The genetic measures $\mathrm{CM}(+)$ and $\mathrm{LDU}(+)$ were not found to be different for chromosome 6 with dense marker spacing. For chromosome 18 with broader marker spacing, the genetic measures based on LDUs were clearly distinguished from the other measures, although they were correlated with $\mathrm{CM}(+)$ (Fig. 2b). LDUs and centimorgans are different measures of genetic distance between markers. LDUs were estimated from the sample of 500 cases and 500 controls and the distances in centi- morgans were provided by Genetic Analysis Workshop 15 (GAW15). Estimates of centimorgans and LDU on a very fine scale require sophisticated methods with assumptions about the coalescence process and may therefore be biased and highly variable. Indeed, we observed that the non-linear relationship of LDUs and centimorgans (Fig. 2a) changed to a linear correlation, when 21 markers from a broader region (mean distances between markers $24 \mathrm{~kb}$ instead of $15 \mathrm{~kb}$ ) around the disease locus on chromosome 6 were considered (data not shown). The resemblance among the different similarity measures based on the MDS analyses was not reflected in the results of the power analysis.

\section{Conclusion}

Based on the results for chromosome 18 using a sample size of 1000, the alternative haplotype similarity measures, except $\mathrm{YU}$, are not superior to the simplest measure, defined as the number of intervals flanked by markers IBS. The observations for Yu's measure suggest that incorporation of marker allele frequencies in the similarity measures may improve the fine mapping properties of haplotype sharing methods. Future work will consider a broader spectrum of genomic regions, including different patterns of physical and genetic marker spacing.

\section{Competing interests}

The author(s) declare that they have no competing interests. 


\section{Acknowledgements}

This work was supported by Deutsche Forschungsgemeinschaft DFG grant CHII7/3-I and BE3906/2-I and the German Bundesministerium für Bildung und Forschung (NGFN grant 0IGR046I).

This article has been published as part of BMC Proceedings Volume I Supplement I, 2007: Genetic Analysis Workshop I5: Gene Expression Analysis and Approaches to Detecting Multiple Functional Loci. The full contents of the supplement are available online at http://www.biomedcentral.com/ $\underline{|753-656| / \mid \text { ? issue=SI. }}$.

\section{References}

I. te Meermann GJ, van der Meulen MA, Sandkuijl LA: Perspectives of identity by descent (IBD) mapping in founder populations. Clin Exp Allergy 1995, 25(Suppl 2):S97-SI02.

2. Beckmann L, Thomas DC, Fischer C, Chang-Claude J: Haplotype sharing analysis using Mantel statistics. Hum Hered 2005, 59:67-78.

3. Morton NE, Zhang W, Taillon-Miller P, Ennis S, Kwok PY, Collins A: The optimal measure of allelic association. Proc Natl Acad Sci USA 200I, 98:5217-522I.

4. Maniatis N, Collins A, Xu CF, McCarthy LC, Hewett DR, Tapper W, Ennis S, Ke X, Morton NE: The first linkage disequilibrium (LD) maps: delineation of hot and cold blocks by diplotype analysis. Proc Natl Acad Sci USA 2002, 99:2228-2233.

5. Yu K, Martin RB, Whittemore AS: Classifying disease chromosomes arising from multiple founders, with application to fine-scale haplotype mapping. Genet Epidemiol 2003, 27: |73-I8I.

6. Cox TF, Cox AA: Multidimensional Scaling Ist edition. London: Chapman \& Hall; 1994.

Publish with Bio Med Central and every scientist can read your work free of charge

"BioMed Central will be the most significant development for disseminating the results of biomedical research in our lifetime. "

Sir Paul Nurse, Cancer Research UK

Your research papers will be:

- available free of charge to the entire biomedical community

- peer reviewed and published immediately upon acceptance

- cited in PubMed and archived on PubMed Central

- yours - you keep the copyright

Submit your manuscript here:

http://www.biomedcentral.com/info/publishing_adv.asp
BioMedcentral 